\title{
Erratum to: RAPD variation in wild, weedy and cultivated azuki beans in Asia
}

\author{
Makiko Mimura $\cdot$ Kentaro Yasuda • \\ Hirofumi Yamaguchi
}

Published online: 21 May 2014

(C) Springer Science+Business Media Dordrecht 2014

\section{Erratum to: Genet Resour Crop Evol 47: 603-610 DOI 10.1023/A:1026584824584}

Unfortunately, the author regrets the original published paper requiring an update, below shown to be read as replacement for the Figs. 1 and 2.
The online version of the original article can be found under doi:10.1023/A:1026584824584.

\section{Mimura}

Department of Bioenvironmental Systems, Tamagawa

University, Machida, Tokyo 194-8610, Japan

e-mail: mimura@agr.tamagawa.ac.jp

\section{K. Yasuda}

Faculty of Bioresource Sciences, Akita Prefectural

University, Ogata, Aktia 010-0451, Japan

e-mail: kentaroy@akita-pu.ac.jp

H. Yamaguchi ( $\square)$

Department of Human and Animal-Plant Relationships,

Tokyo University of Agriculture, Atsugi,

Kanagawa 243-0034, Japan

e-mail: h4yamagu@nodai.ac.jp

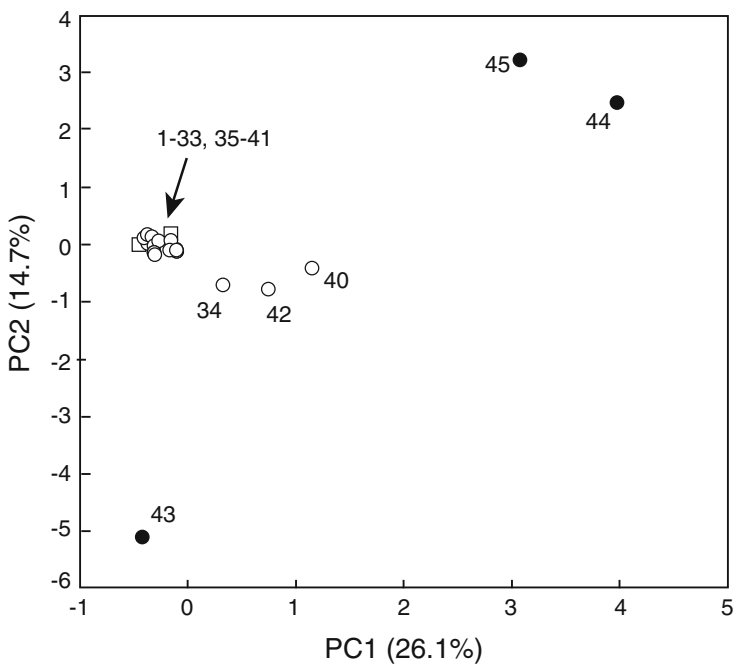

Fig. 1 Representation of the three races of azuki beans (accessions 1-42) and two outgroup species (accessions 43, 44 , and 45) on the first and second principal components. The axes are given with their percentage to the contribution of total variation. Cultivated azuki accessions are represented by open squares, weedy azuki bean by open hexagons, and wild azuki bean by open circles. Numbers indicate accession codes (see Table 1). Accession number 43 is V. nakashimae, and 44 and 45 are $V$. umbellata as outgroups 


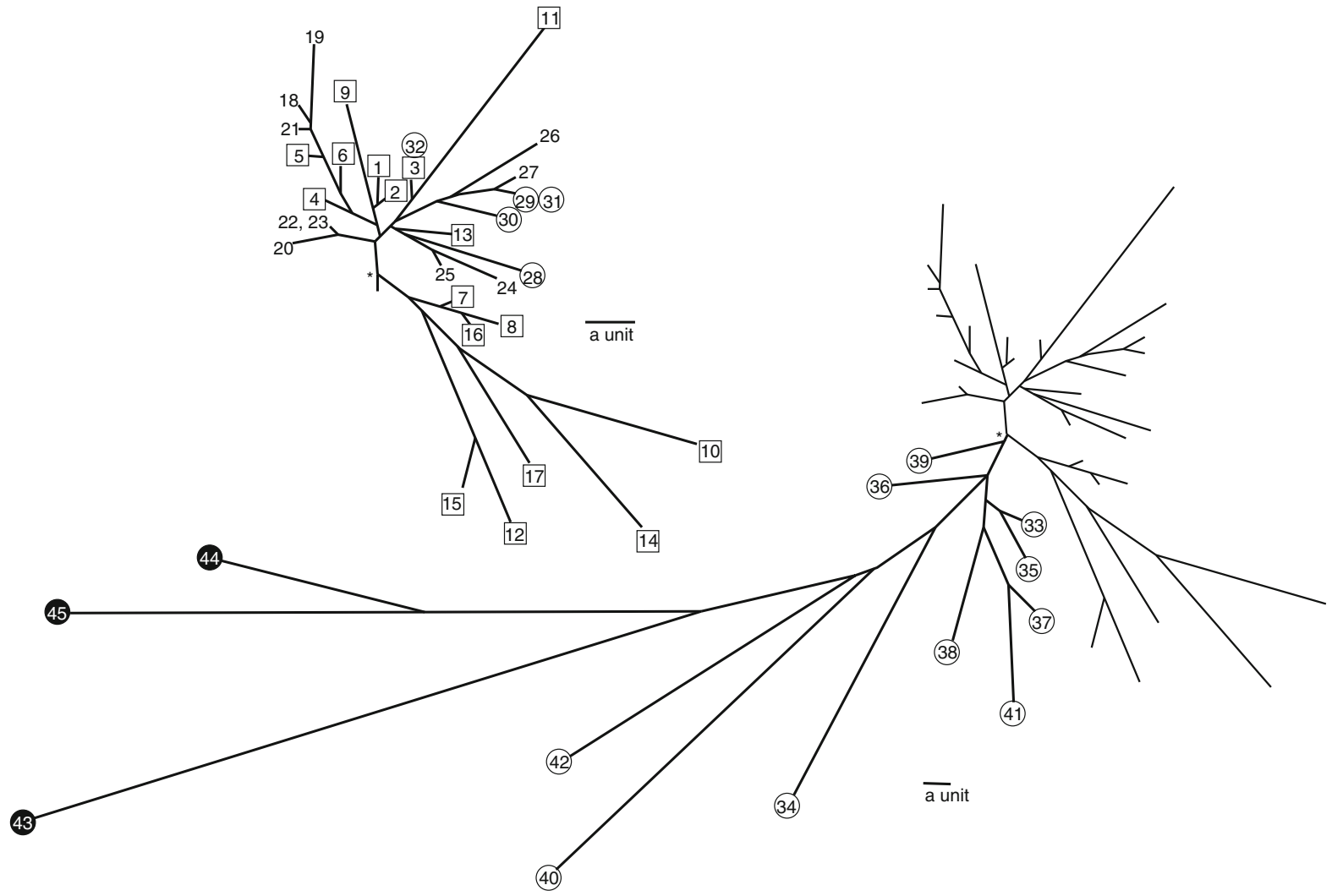

Fig. 2 Genetic relationship among 42 accessions of azuki beans and two outgroup species by the neighbour-joining (NJ) method. Cultivated azuki accessions are represented by open squares, weedy azuki bean without symbol, and wild azuki bean by open circles. Numbers indicate accession codes (see
Table 1). Accession number 43 is V. nakashimae, and 44 and 45 are $V$. umbellata as outgroups. The horizontal bar shows one unit, 0.01 point, of distance by the NJ method. The densely aggregated network in upper right portion of the whole network is shown in the upper left 